Copyright (@ 2009 Institute of Electrical and electronics Engineers, Inc.

All Rights reserved.

Personal use of this material, including one hard copy reproduction, is permitted.

Permission to reprint, republish and/or distribute this material in whole or in part for any other purposes must be obtained from the IEEE.

For information on obtaining permission, send an e-mail message to stds-igr@ieee.org.

By choosing to view this document, you agree to all provisions of the copyright laws protecting it.

Individual documents posted on this site may carry slightly different copyright restrictions.

For specific document information, check the copyright notice at the beginning of each document. 


\title{
An Advanced Optimal Approach for High Voltage AC Bushing Design
}

\author{
Mohammad R. Hesamzadeh, Nasser Hosseinzadeh and Peter Wolfs \\ Central Queensland University \\ Power Engineering Group \\ Rockhampton, QLD, Australia
}

\begin{abstract}
This paper proposes a new and advanced methodology for finding the optimum electrical design of high voltage ac capacitive graded bushings using an improved genetic algorithm approach as an effective meta-heuristic method. A case study has been conducted on a $145 \mathrm{kV}$ oil impregnated paper (OIP) bushing and the IEC 60137 tests have been performed to evaluate its performance. Condenser-bushings contain concentric conductive foils which are isolated from each other. The partial capacitances between conducting cylinders can be modified by adjusting the number, diameter, place and length of these cylinders as well as the thickness of insulating material between foils. As a result, the voltage drop and also the electrical stress in the core and along the surface will change. This paper finds optimal value of bushing design parameters to achieve well-distributed electric stress with the lowest possible maximum value and also a constant voltage drop for different layers by using an improved genetic algorithm optimization method subject to practical and technological constrains. The proposed method of this research work has been applied to a $145 \mathrm{kV}$ OIP bushing. The performance of optimal designed $145 \mathrm{kV}$ OIP bushing under IEC 60137 tests is very promising.
\end{abstract}

Index Terms - High voltage techniques, modified genetic algorithm, insulators, dielectric breakdown, IEC.

\section{INTRODUCTION}

IN today's competitive market, there is a need for the bushing manufacturing industry to improve bushing efficiency and to reduce costs; because high-quality low-cost products and processes have become the key to survival in the global economy. On the other hand, with the increase of electrical energy demand, the voltage levels of electric transmission systems have increased rapidly within recent years. The reliability of equipment and facilities used in a power system is an essential precondition of the energy transmission security. Bushing failure is one of the leading causes of transformer failures $[1,2]$.

Researchers have reported various aspects of high voltage bushing design in the literature. In [3], Lachman et al proposed the application of the sum current method to on-line diagnostics of high-voltage bushings and current transformers. Guoqiang et al applied evolution strategy for optimal design of high voltage bushing electrode in transformers [4]. In [5], Lampe from Swedish Transmission Research Institute reported the measured electric field along HVDC bushings

Manuscript received on 13 April 2007, in final form 15 August 2007. with hydrophobic surfaces under different environmental conditions. Significantly reduced radial field strength was found for the hydrophobic bushing. Laboratory tests to evaluate HVDC wall bushings performance in wet weather was investigated by Lambeth in [6]. Finding an accurate model for capacitive graded bushings for fast transient overvoltages in GIS was discussed by Ardito [7]. Zhu Fang proposed a new method for finding the optimum design of lower part of bushing in order to decrease the flashover voltage in oil and the partial inception voltage (PDIV) [8]. Salam proposed a method for optimizing the field stress on HV bushings by modifying their profile, seeking a uniform distribution of the tangential field along the bushing surface [9]. Capacitive-resistive field calculation on HV bushings using the boundary-element method was proposed by Chakravort [10]. Monga et al published a paper [11], which illustrated the use of electric field computations to optimize the design of gas filled high voltage composite bushings.

To our knowledge and considering the above literature review, the optimal electrical design of condenser-graded high voltage ac bushings has not been reported, although it is one of the leading causes of bushing failures. That is, from an electrical point of view, a poorly designed bushing may face 
many problems like partial discharge, flashover, and deterioration of bushing insulation during tests or operation in a power system network. In addition, having an optimum electrical design for bushings can lead to minimum usage of materials along with longer life time and higher reliability that are important factors for bushing manufacturers. Therefore, it has been the theme of this research work to establish an approach for the optimal electrical design of high voltage ac bushings in order to minimize the catastrophic failures of bushings and guarantee the longer period of operation.

This paper has been organized in five sections including this introduction. In Section 2, mathematical model of the problem, which includes design parameters, suggested indices for assessment of performance of a designed bushing, technological and manufacturing restrictions of each parameter have been developed. The mathematical model has been formulated as an optimization problem and the paper uses the improved genetic algorithm optimization method for finding the optimum electrical design.

In Section 3, a $145 \mathrm{kV}$ oil-impregnated paper (OIP) bushing has been designed using advanced optimal approach proposed by this paper and then manufactured. Typical and routine tests according to the IEC 60137 standard have been carried out on the manufactured optimally designed bushing. The performance indices of this optimum design have been compared with the indices of a conventionally designed bushing, which demonstrates a considerable improvement in performance indices of optimally designed bushings. Finally, a brief discussion about the results of typical and routine tests which have been performed on the optimally designed manufactured bushing closes the paper.

\section{MATHEMATICAL MODEL OF THE PROBLEM}

The electrical design of capacitive grading bushings is one of the important parts of manufacturing of these kinds of bushings. In this step, engineers design the condenser bushings with practical design parameters and with considering the technological constrains. It goes without saying that finding an optimum design has its own importance in this stage. The reason is that it leads to using minimum amount of raw materials and lower cost of manufacturing process along with higher performance of condenser bushings during operation on power transformers. These are very important factors for manufacturing companies.

Capacitive grading bushings contain embedded in their insulation core concentric conductive foils, which are isolated from each other. By adjusting the diameter and length of these cylinders, the electrical stress and voltage drop in the core and along its surface can be influenced by variation of the ratio of the partial capacitances between the conducting cylinders. Because of some manufacturing limitations, this paper suggests the use of the foil configuration shown in Figure 1.

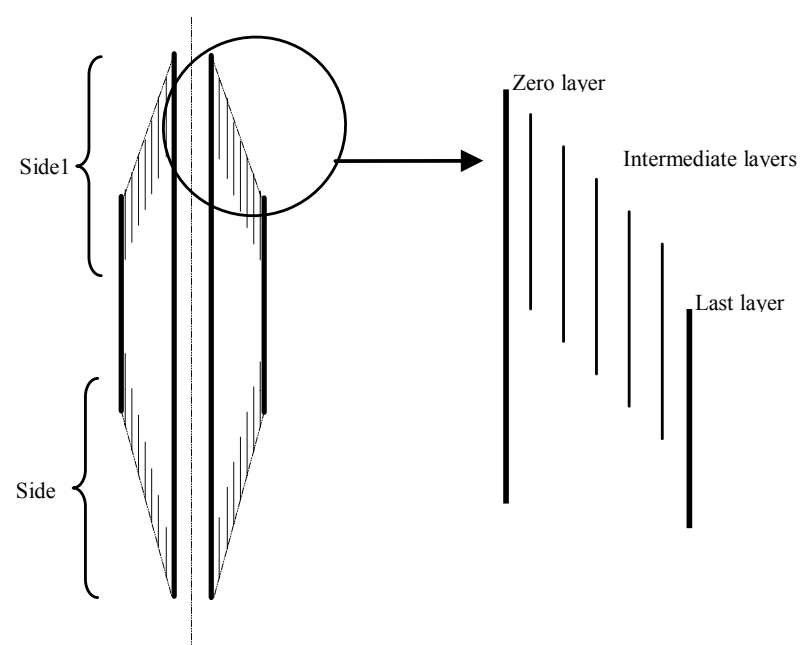

Figure 1. Conic-TYP2 foil configuration.

The grading of ac-bushing is achieved from the capacitances that are formed between the grading foils and thus determined by the permittivity of the insulating material. The electrical field can be calculated from equation (1).

$$
\begin{aligned}
& \oint \overrightarrow{\mathrm{E}} \cdot \mathrm{d} \dot{\mathrm{l}}=0, \oint \overrightarrow{\mathrm{D}} \cdot \mathrm{d} \overrightarrow{\mathrm{A}}=\mathrm{Q} \text { and } \overrightarrow{\mathrm{D}}=\varepsilon \overrightarrow{\mathrm{E}} \\
& \overrightarrow{\mathrm{E}}: \text { Vector of electrical stress } \\
& \mathrm{d} \dot{\mathrm{l}} \text { : Vector of element of integration path } \\
& \mathrm{d} \overrightarrow{\mathrm{A}}: \text { Vector of elemement of area } \\
& \overrightarrow{\mathrm{D}}: \text { Vector of electrical flux density } \\
& \varepsilon: \text { permittivity }
\end{aligned}
$$

The conic-TYP2 foil configuration uses cylinder capacitors. Voltage drop on each layer can be found by the series-parallel configuration of these cylinder capacitors.

Optimum condenser bushing designs can be formulated as an optimization problem with relevant restrictions. The experience of authors in the design of condenser bushings shows that a suitable objective function along with practical restrictions can be introduced as follows:

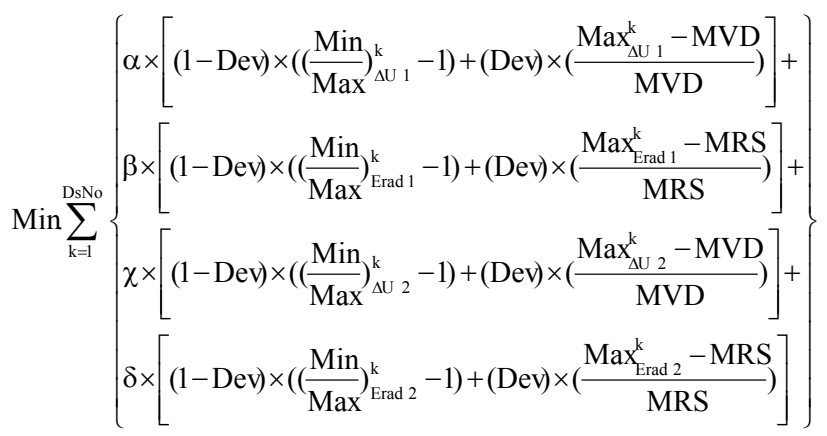

Such that:

1. $\varepsilon_{\text {Min }} \leq \varepsilon \leq \varepsilon_{\text {Max }}$

2. $\mathrm{Nfl}_{\text {Min }} \leq \mathrm{Nfl} \leq \mathrm{Nfl}_{\text {Max }}$

3. Dint $_{\text {Min }} \leq \operatorname{Dint} \leq$ Dint $_{\text {Max }}$ 

4. Dext $_{\text {Min }} \leq$ Dext $\leq$ Dext $_{\text {Max }}$
5. $\mathrm{Lx}_{\mathrm{Min}} \leq \mathrm{Lx} \leq \mathrm{Lx}_{\mathrm{Max}}$
6. $\mathrm{Lpl}_{\mathrm{Min}} \leq \mathrm{Lpl} \leq \mathrm{Lpl}_{\mathrm{Max}}$
7. $\mathrm{Sp} 1_{\mathrm{Min}} \leq \mathrm{Sp} 1 \leq \mathrm{Sp} 1_{\text {Max }}$
8. $\mathrm{Lp} 2_{\text {Min }} \leq \mathrm{Lp} 2 \leq \mathrm{Lp} 2_{\text {Max }}$
9. $\mathrm{Sp} 2_{\mathrm{Min}} \leq \mathrm{Sp} 2 \leq \mathrm{Sp} 2_{\text {Max }}$
10. $\Delta \mathrm{U} \leq \mathrm{MVD}$
11. Eaxl $\leq$ MAS
12. Foils Configuration Must Be Conic-TYP2
13. $\alpha+\beta+\chi+\delta=1$

The objective function given in equation 2 and its restrictions as given in equation 3 are explained as follows. The insulation of a capacitive grading bushing is stressed, as shown in Figure 2, radially and axially. Any area above the boundary surface between the insulating material and surrounding medium should be considered as a critical area.

The radial component of the electric field strength can cause serious breakdown of the insulating material, whilst under certain circumstances, the axial component can lead to surface discharges along the boundary surface. Since the electric strength of the insulating material stressed to breakdown limit is appreciably higher than that of the boundary layer stressed to flashover limit, the axial stress is in general far more critical.

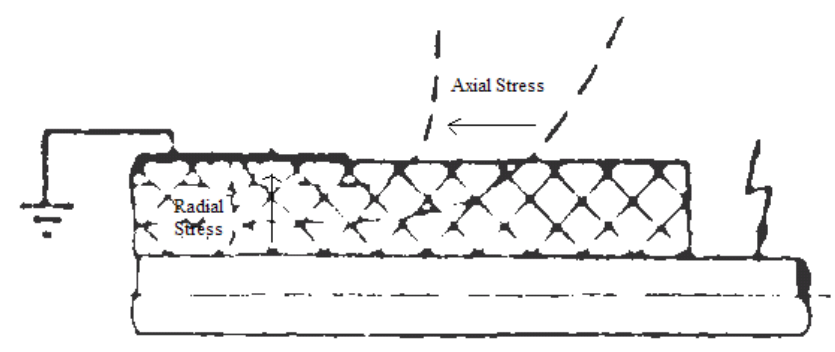

Figure 2. Radial And Axial Stress in high voltage bushings.

Based on this fact, this paper includes the radial component of electric strength in the objective function introduced earlier and the minimization is done only on this component of the electric strength. The axial component of the electrical strength has been considered as a constraint of the objective function and checked only according to its maximum value.

In the objective function, introduced by equation (2), there are two terms for each radial component of electrical strength that relates to each other through the Dev variable. Dev as an integer variable, which can only be either 0 or 1 according to the value of the corresponding designed parameter. For each design, the radial electrical stress is calculated and if this value is more than the maximum value, the $D e v$ variable takes 1 and the minimization is done on $\left(\frac{M a x_{E r a d}^{k}-M R S}{M R S}\right)$ term. In this term, $\operatorname{Max}_{E r a d 1}^{k}$ is the maximum value of radial stress in the side number 1 of the condenser bushing for design number $\mathrm{k}$ (total number of designs is $D s N o$ ), and also $M R S$ introduces the
Maximum Radial Stress. In this process, when $\operatorname{Max}_{E r a d 1}^{k}$ becomes lower than $M R S$, then the Dev variable takes zero value and the minimization process is done on the $\left.\left(\frac{M i n}{M a x}\right)_{E r a d 1}^{k}-1\right)$ term. From that point, the minimization process attempts to make the maximum radial stress on side 1 ( Max ${\stackrel{E r a d}{k}{ }_{1}}_{\text {) }}$ ) equal to the minimum amount of it ( Min ${ }_{\text {Erad }}^{k}$ ). The reason is that the best exploitation of the insulating material in view of its insulating strength is achieved when the radial stress is kept constant. However, it should be noted that this concept can not be achieved in practical cases. But, this process is attempted in the design phase to achieve an optimum solution. In this paper, this process is embedded in its suggested objective function.

In addition, for optimum utilization of the dielectric, it is recommended that the capacitive grading be arranged so that the same partial voltage is across two adjacent layers [12]. To meet this condition, similar to radial electrical stress, the objective function includes two terms for representing voltage drop on each layer. These terms relate to each other by $D e v$ variable that can accept 0 or 1 . The $\left(\frac{M a x_{\Delta U 1}^{k}-M V D}{M V D}\right)$ term minimizes the maximum voltage drop on each layer to an amount that is lower than the permitted value, that is the parameter $M V D$ in equations (2) and (3). When maximum voltage drop for different layers ( $M a x_{\Delta U 1}^{k}$ ) becomes lower than the permitted value, Dev variable gets a 0 value and the objective function minimizes the term $\left.\left(\frac{\operatorname{Min}}{\operatorname{Max}}\right)_{\Delta U 1}^{k}-1\right)$, so that the minimum and maximum voltage drop reach to an equal amount.

In the objective function of equation (2), index 1 relates to side 1 of the condenser bushing and index 2 relates to side 2 of the condenser bushing. $\alpha, \beta, \chi$, and $\delta$ are weight factors of different design parameters, namely, radial electrical stress and voltage drop on each layer of sides 1 and 2. These factors are normally chosen by the bushing design engineer based on the empirical rules.

With Regard to constraints 1 to 12 , $\varepsilon$ (circle 1 in Figure 3 ) shows the permittivity of insulating material that can have a maximum and minimum value according to available insulating materials. $\mathrm{Nfl}$ describes the number of foils that can be used for making partial cylinder capacitors in condenser bushing and it can vary between two margins according to the experience of the design engineer. Dint (circle 2 in Figure 3) and Dext (circle 3 in Figure 3 ) are the diameter of high voltage conductor and inner diameter of outer porcelain insulator. These two parameters can have minimum and maximum values according to the current level of bushing and the required volume of oil for removing heat caused by the passing current. $L x$ (circle 4 in Figure 3), Lpl (circle 5 in Figure 3), and $L p 2$ (circle 6 in Figure 3) are zero layer foil length, intermediate layer foil length of side 1 and 2. The horizontal distances between intermediate foil layers are included by $S p 1$ (circle 7 in Figure 3) and $S p 2$ (circle 8 in 
Figure 3). $D U$ is the voltage drop on each partial capacitor created by partial foils and Eaxl is the axial component of radial stress. The maximum permitted values of these variables are $M V D$ (maximum voltage drop) and $M A S$ (maximum axial stress). Finally, the constraint number 12 points to the Conic-TYP2 configuration of foils (Figure 1). The design parameters are illustrated in Figure 3.

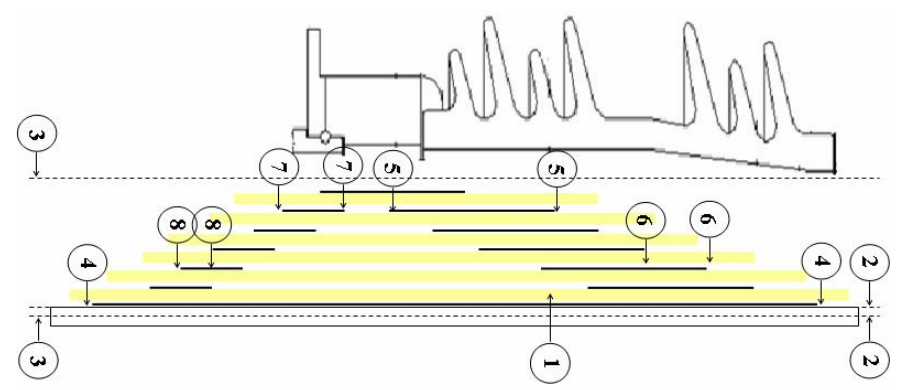

Figure 3. Design parameters of capacitive grading bushings

The Genetic Algorithm (GA) as a meta-heuristic optimization methodology is proposed to solve the optimal bushing design problem. The main idea of GA is that "the best member of a population has the highest probability for survival and reproduction" [13], [14]. Tools applying GA are reported in the literature to be capable of finding a global optimum for mathematical problems having a multiplicity of local optimum and hard non-convexities. GA has also proved powerful in the optimization process in various power engineering applications, e.g. [13], [15] and [16]. The improved genetic optimization algorithm, as applied to optimum bushing design, observes the following steps:

Decision variables in GA are the nine variables as introduced in constrains of 1 to 9 of equation set (3). A typical chromosome is shown in Table 1.

Table 1. Chromosome structure for different designs.

\begin{tabular}{|l|l|l|l|l|l|l|l|l|}
\hline $\boldsymbol{\varepsilon}$ & Nfl & Dint & Dext & Lx & Lp1 & Sp1 & Lp2 & Sp2 \\
\hline
\end{tabular}

The GA requires the definition of an initial population. As previously mentioned, each member of the population in the case of this paper is an individual design of the condenser bushing. For in-feeding practical experiences in the design of the bushing, the first generation of genetic algorithm intakes the practical available design parameters of a similar bushing.

The well known operators for genetic algorithm, namely, crossover and mutation, as explained in the literature on genetic algorithm theory $[16,17]$ are used in this paper, too.

Selecting the best available practically designed bushing parameters as the original population, the population grows through the addition of new members, which are obtained from the crossover and mutation steps. This enlarged

Fitness $\left(w_{i}\right)=\left\{\begin{array}{l}\operatorname{Obj} \operatorname{Val}\left(w_{i}\right) \text { If } w_{i} \text { meets all constrains } \\ B \quad \text { If } w_{i} \text { doesnot meet all constrains }\end{array}\right.$ population is ranked with a fitness function defined as follows: $\mathrm{w}_{\mathrm{i}}$ : A sample chromosome

B: A large number

Obj Val $\left(w_{i}\right)$ : Object value for chromosome $w_{i}$
It means that if a design satisfies all constraints in equation set (2), then the objective function for that design will be determined; otherwise, a large number will be assigned to that design as its fitness.

A reduction of the enlarged population is made using the ranking in order to maintain the original population size. A new generation is then determined as a mixture of some members of the previous population and some new members resulting from the crossover and mutation steps. Poor designs, which do not satisfy all constrains will be eliminated.

In the following section, the optimum electrical design of a typical bushing is proposed.

\section{CASE STUDY (BUSHING 145KV-OIP)}

In order to implement the proposed method, a software package has been developed.

The basic technical specifications of the oil impregnated paper bushing chosen for the case design are as follows:

Highest voltage for equipment $\left(\mathrm{U}_{\mathrm{m}}\right)$

$145 \mathrm{kV}$

Rated frequency

Rated power frequency withstand voltage

Rated current

$50 \mathrm{~Hz}$

$275 \mathrm{kV}$

$800 \mathrm{~A}$

The practical data for minimum and maximum values of design parameters, as well as design constrains as introduced in equation (2), has been collected in Table2.

\begin{tabular}{l|cc}
\multicolumn{3}{l}{ Table2. Maximum and minimum values of bushing design parameters. } \\
\hline Design parameter & Minimum & Maximum \\
\hline$\varepsilon$ of Oil Impregnated Paper (10-3F/m) & 0.0283 & 0.0483 \\
No. of foils & 20 & 80 \\
Dint(mm) & 40 & 45 \\
Dext(mm) & 100 & 105 \\
Zero layer length, Lx(mm) & 1200 & 1500 \\
Length of partial foils in side 1, Lp1 & 200 & 500 \\
(mm) & & \\
Length of partial foils in side 2, Lp2 & 100 & 300 \\
$(\mathrm{~mm})$ & & \\
Length of steps in side1,Sp1 & 10 & 40 \\
(mm) & & \\
Length of steps in side2,Sp2 & 10 & 40 \\
(mm) & - & 3.5 \\
MVD(kV) & - & 5.2 \\
MRS(kV/mm) & - & 0.4 \\
MAS $(\mathrm{kV} / \mathrm{mm})$ &
\end{tabular}

Table 3 includes GA basic settings in running the developed program for finding the optimum design of bushing.

Table 3. Values of Genetic Algorithm settings.

\begin{tabular}{c|c} 
GA parameter & Value \\
\hline Population size & 300 \\
ross over probability & 0.9 \\
Mutation probability & 0.3 \\
Ending criterion & 100 \\
$\alpha$ (p.u.) & 0.4 \\
$\beta$ (p.u.) & 0.1 \\
$\chi$ (p.u.) & 0.4 \\
$\delta$ (p.u.) & 0.1 \\
\hline
\end{tabular}


The results of optimal design of this bushing and also a conventional design are given in Tables 4 and 5 .

Table 4. Decision variables using a conventional design and an optimum design by genetic algorithm.

\begin{tabular}{c|ccccc}
\hline & Epsilon & Nfl & Dint & Dext & Lx \\
\hline Conventional & 0.0283 & 22 & 42 & 106 & 1300 \\
Optimum & 0.0283 & 26 & 40 & 104 & 1497 \\
& Lp1 & Sp1 & Lp2 & Sp2 & \\
Conventional & 315 & 30 & 130 & 15 & \\
Optimum & 305 & 15 & 197 & 10 & \\
\hline
\end{tabular}

Table 5. Percentage of improvement of performance indices (objective value) using a conventional design and also an optimum design by improved genetic algorithm.

\begin{tabular}{|c|c|c|c|c|}
\hline & & $(\operatorname{Min} / \operatorname{Max}-1)$ & Average & STD \\
\hline \multirow{3}{*}{ Erad1 } & Conventional & 0.42 & 2.67 & 0.45 \\
\hline & Optimum & 0.08 & 2.66 & 0.07 \\
\hline & $\begin{array}{c}\% \\
\text { improvement }\end{array}$ & 80.95 & 0.37 & 84.44 \\
\hline \multirow{3}{*}{ Erad2 } & Conventional & 0.54 & 2.64 & 0.62 \\
\hline & Optimum & 0.10 & 2.62 & 0.09 \\
\hline & $\begin{array}{c}\% \\
\text { improvement }\end{array}$ & 81.48 & 0.75 & 85.48 \\
\hline \multirow{3}{*}{ DU1 } & Conventional & 0.43 & 3.81 & 0.66 \\
\hline & Optimum & 0.09 & 3.22 & 0.10 \\
\hline & $\begin{array}{c}\% \\
\text { improvement }\end{array}$ & 79.07 & 15.48 & 84.84 \\
\hline \multirow{3}{*}{ DU2 } & Conventional & 0.55 & 3.81 & 0.91 \\
\hline & Optimum & 0.11 & 3.22 & 0.12 \\
\hline & $\begin{array}{c}\% \\
\text { improvement }\end{array}$ & 80 & 15.48 & 86.81 \\
\hline
\end{tabular}

\begin{tabular}{cc}
\hline Erad1(2) & Maximum Radial Stress in Different Layers of s \\
& $1(2)$ \\
DU1(2) & Voltage Drop on Different Layers of Side \\
STD & $1(2)$ \\
$\%$ & Standard Deviation \\
improvement & Conventional Value - Optimum Value $\mid /$ \\
\end{tabular}

It is clear from Table 5 that the proposed method is very promising with due consideration to the essential technological limitations.

In the case of a (Min/Max - 1) index, the Erad1 has an improvement of $80.95 \%$, Erad2 an improvement of $81.48 \%$, DU1 an improvement of $79.07 \%$, and DU2 an improvement of $80 \%$. With regard to the average, one can see $0.37 \%, 0.75 \%$, $15.48 \%$, and $15.48 \%$ improvement in Erad1, Erad2, DU1, and DU2, accordingly. By comparing the standard deviation of Erad1, Erad2, DU1, and DU2, it is obvious that in the case of optimum design the deviation of data from the average has decreased considerably. The improvement for the standard deviation is $84.44 \%$ for Erad1, 85.48\% for Erad2, 84.84\% for DU1, and finally $86.81 \%$ for DU1.

For easier comparison, Figure 4 shows maximum radial electrical stress for each capacitive layer and Figure 5 shows voltage drop on each capacitive layer in both conventional and optimum design. According to these figures, a nearly constant electrical radial stress and also voltage drop (in the case of different layers) have been achieved.

The measured results of the dielectric dissipation factor and the capacitance of the optimally designed bushing compared with the conventionally designed one showed a promising improvement of $51 \%$ in tangent of delta value and also satisfied the clause 9.1 of IEC60137.

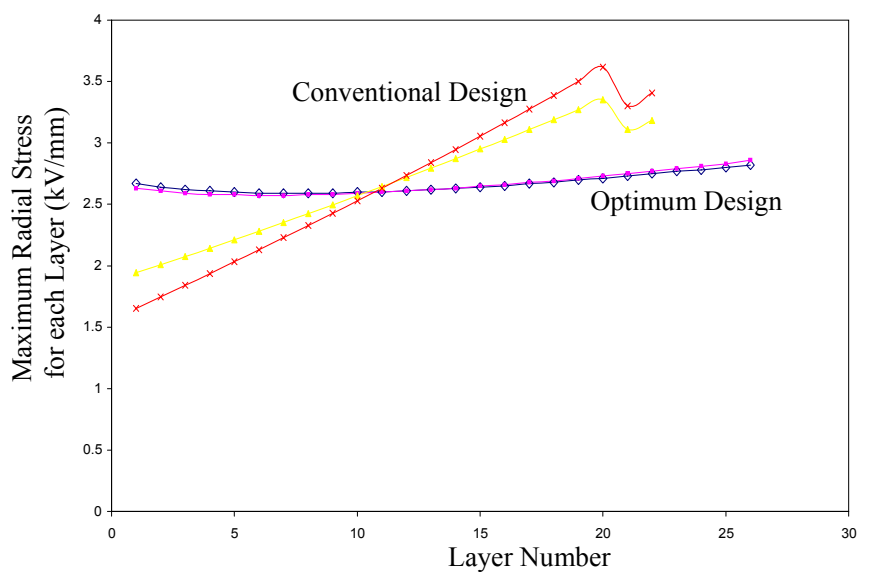

Figure 4. Maximum radial electrical stresses for each capacitive layer for conventional and capacitive layer for conventional and optimum design

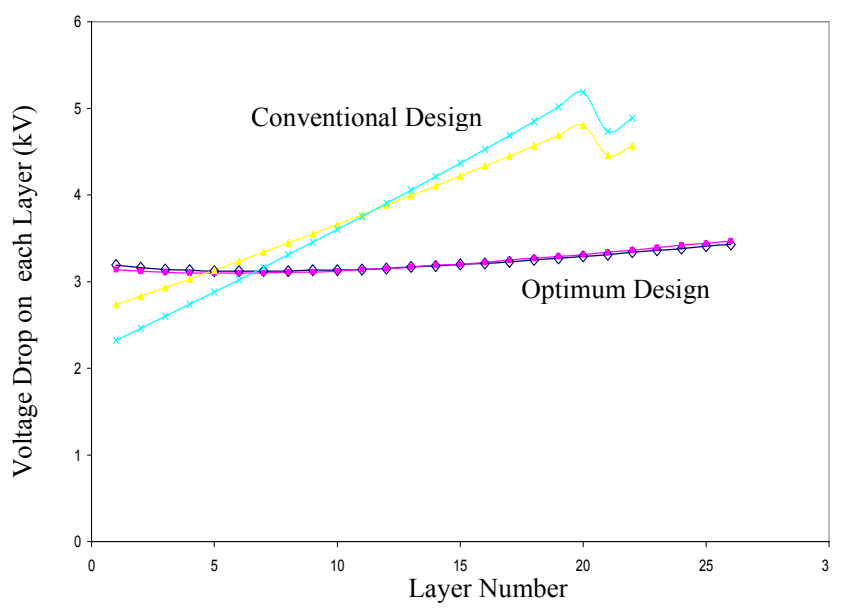

Figure 5. Voltage drop on each capacitive layer for conventional and optimum design.

For the case of optimally designed bushing at applied voltages of $13 \mathrm{kV}, 50 \mathrm{kV}, 88 \mathrm{kV}\left(1.05 \mathrm{U}_{m} / \sqrt{3}\right), 145 \mathrm{kV}\left(\mathrm{U}_{\mathrm{m}}\right), 88 \mathrm{kV}$ $\left(1.05 U_{m} / \sqrt{3}\right), 50 \mathrm{kV}$, and $13 \mathrm{kV}$, the average measured capacitance was $208.8 \mathrm{pF}$ with the standard deviation of 0.06 . Also, the average measured value of tangent of delta was $24.15 \times 10^{-4}$ with the standard deviation of 0.11 .

Measurement of partial discharge quality of the optimally designed bushing showed a partial discharge level of $4 \mathrm{pC}$ at the applied voltage of $145 \mathrm{kV}$ for $10 \mathrm{~s}$, and smaller than $1 \mathrm{pC}$ at the applied voltages of $126 \mathrm{kV}\left(1.5 U_{m} / \sqrt{3}\right)$ and $88 \mathrm{kV}$ $\left(1.05 \mathrm{U}_{\mathrm{m}} / \sqrt{3}\right)$ for $10 \mathrm{~s}$, which readily satisfy the IEC60137 
clause 9.4. Compared with the conventionally designed bushing, results of the partial discharge for optimally designed case had an improvement of 50\% in the worst case of applied voltage of $145 \mathrm{kV}$.

Finally, with regard to the dry power-frequency voltage withstand test, the optimally designed bushing passed the applied voltage of $275 \mathrm{kV}$ with frequency of $50 \mathrm{~Hz}$ and duration of $60 \mathrm{~s}$ without any flashover or breakdown, which satisfies clause 9.3 of IEC60137.

\section{CONCLUSION}

High voltage bushing breakdown is one of the major contributors to the transformer failures. Since the electrical design of the HV bushings is the most important part of their manufacturing process, finding an algorithm for the electrical design of bushings in an optimum way is very important.

This paper proposes an advanced method for finding optimum electrical design of capacitive grading bushings. The proposed method finds the best values of decision variables (parameters) in the design of a capacitive grading bushing according to a technological objective function with its corresponding constraints. This is done by using an improved genetic algorithm (GA) as a powerful meta-heuristic optimization method. For in-feeding the practical experiences, the GA starts from a set of available design parameters for similar bushings. Promising performance indices and laboratory test results were obtained by applying this method to a typical $145 \mathrm{kV}$ oil impregnated paper bushing.

\section{REFERENCES}

[1] V. Smekalov, "Bushing insulation monitoring in the course of operation", Transaction in CIGRE proceedings, 12-106, 1996.

[2] S. D. Kassihin, S.D. Lizunov, G.R. Lipstein, A.K. Lokhanin, and T. I. Morozova, "Service experience and reasons of bushing failures of EHV transformers and shunt reactors", Trans. CIGRE proceedings, pp. 12$105,1996$.

[3] M. F. Lachman, W. Walter, and P.A. Guggenberg, "On-line diagnostics of high-voltage bushings and current transformers using the sum current method", IEEE Trans. Power Delivery, Vol. 13, pp. 155-162, 2000.

[4] Z. Guoqiang, Z. Yuanlu, and C. Xiang, "Optimal design of high voltage bushing electrode in transformer with evolution strategy", IEEE Trans. Magnetics, Vol. 15, pp. 1690-1693, 1999.

[5] W. Lampe, D. Wikstrom, and B. Jacobson, "Field distribution on an HVDC wall bushing during laboratory rain tests", IEEE Trans. Power Delivery, Vol. 6, pp. 1531-1540, 1991.

[6] P.J. Lambeth, "Laboratory tests to evaluate HVDC wall bushing performance in wet weather", IEEE Trans. Power Delivery, Vol. 5, pp. 1782-1793, 1990.

[7] A. Ardito, R. Iorio, G. Santagostino, and A. Porrino, "Accurate modeling of capacitively graded bushings for calculation of fast transient overvoltages in GIS", IEEE Trans. Power Delivery, Vol. 7, pp. 13161327, 1992.

[8] Z. Fang, J. Jicun and Z. Ziyu, "Optimal design of HV transformer bushing", $3^{\text {rd }}$ Intern. Conf. Properties and Applications of Dielectric Materials, Tokyo, Japan, pp. 434-437, 1991.
[9] M. Abdel-Salam and A. Mufti, "Optimizing field stress on high voltage bushings", IEEE Intern. Sympos. Electr. Insul. (ISEI), Pittsburg, PA, USA, pp. 225-228, 1994.

[10] D. Kind and H. Karner, High-Voltage Insulation Technology, Friedrick Vieweg \& Son, August 1985.

[11] S. Monga and R. S. Gorur, "Design optimization of high voltage bushing using electric field computations", IEEE Trans. Dielectr. Electr. Insul., Vol. 13, pp. 1217-1224, 2006.

[12] S. Chakravorti and H. Steinbigler, "Capacitive-resistive field calculation on HV bushings using the boundary-element method", IEEE Trans. Dielectr. Electr. Insul., Vol.5, pp. 237-244, 1998.

[13] A. S. Chung, and F. Wu, "An extensile genetic algorithm framework for problem solving in a common environment", IEEE Trans. Power Systems, Vol. 15, pp. 269-275,.2000.

[14] J. H. Holland, "Genetic algorithm", Scientific American, Vol. 267, No. 1, pp. 66-73, 1992.

[15] D. E. Goldberg, Genetic Algorithms in Search, Optimization, and Machine Learning, Addison Wesley, 1989.

[16] T. Yokota, M. Gen, and L. Yin-Xin, "Genetic algorithm for non-linear mixed integer programming problems and its applications", Elsevier, Computers and. Industrial Engineering, Vol. 30, pp. 905, Sep. 1996.

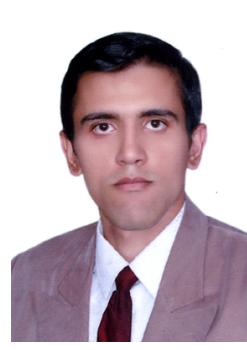

engineering.
Mohammad R. Hesamzadeh (S'07) was born in Shiraz, Iran in 1980. He graduated from Shiraz University in 2002 with a B.Eng. degree and received the M.Eng. degree from Tarbiat Modarres University in 2004. He has worked as a researcher at industrial companies and research centers in the areas of power system and high voltage engineering for 4 years. Currently, he is a Ph.D. student at Central Queensland University in Australia. His special fields of interest include power system restructuring, high voltage engineering and intelligent system applications in

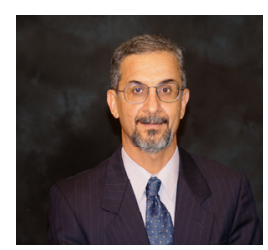

Nasser Hosseinzadeh (M'86) was born in Iran in 1960. He graduated from Shiraz University in 1986 with a B.Sc. degree in electrical and electronics engineering. He worked in a research centre for five years before starting his postgraduate studies. He received a M.Sc. degree from Iran University of Science and Technology in 1992 and a Ph.D. degree from Victoria University in Australia in 1998. Dr Hosseinzadeh is currently the Head of Department of Systems at the Faculty of Sciences, Engineering and Health at Central Queensland University, Rockhampton, Australia. His special fields of interest include power system analysis and planning, power system stability, intelligent system applications in engineering, distributed generation and rural energy supply.

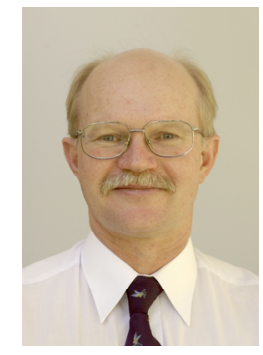

Peter Wolfs (M'80-SM'99) was born in Rockhampton Australia in 1959. He graduated from the Capricornia Institute of Advanced Education in 1980 with a B.Eng. degree. He subsequently obtained the M.Eng. degree from the Philips International Institute in the Netherlands in 1981 and the Ph.D. degree at the University of Queensland in 1992. He is the Associate Dean (Research and Innovation) at the Faculty of Sciences, Engineering and Health at Central Queensland University. His special fields of interest include rural and renewable energy supply, solar and hybrid electric vehicles and intelligent systems applications in railways. Professor Wolfs is a Fellow of Engineers Australia, a Registered Professional Engineer in the State of Queensland and a member of the Railway Technical Society of Australia. 